\title{
Brain Mapping: Real-Time Neuropsychological Testing Experience during Low-Grade Tumor Resection
}

\author{
Barbara Tomasino $^{1} \bullet$ Tamara lus $^{2} \bullet$ Miran Skrap² $^{2}$ \\ ${ }^{1}$ Scientific Institute IRCCS "Eugenio Medea", Polo FVG, San Vito al Tagliamento (PN), \\ Italy; ${ }^{2}$ Unità Operativa di Neurochirurgia, Azienda Sanitaria Universitaria del Friuli \\ Centrale S. Maria della Misericordia, Udine, Italy
}

Author for correspondence: Barbara Tomasino, IRCCS "E. Medea", Polo Regionale del Friuli Venezia Giulia, Via della Bontà, 7, San Vito al Tagliamento (PN), 33078, Italy.

E-mail: barbara.tomasino@lanostrafamiglia.it

Doi: https://doi.org/10.36255/exonpublications.gliomas.2021.chapter11

\begin{abstract}
Awake surgery and direct electrical stimulation are performed to maximize the extent of resection while minimizing the risk of neurological and cognitive deficits. Direct electrical stimulation is a highly reliable method for monitoring simple brain functions when stimulating the sensorimotor cortex, or areas involved in speech articulation; however, negative mapping increases when testing higher functions related to language or cognition. By using DES alone, when resection involves areas supporting higher level cognition, the surgeon may receive poor feedback on the patient's cognitive status. To collect more information on the patient's cognitive status during resection, we developed real-time neuropsychological testing, an intensive neuropsychological monitoring method which is performed in addition to direct electrical stimulation. The technique includes a large number of tests that are administered in a continuously rotating and repeating pattern at different stages per anatomical area. The aim is to have a
\end{abstract}

In: Gliomas. Debinski W (Editor). Exon Publications, Brisbane, Australia. ISBN: 978-0-6450017-4-7; Doi: https://doi.org/10.36255/exonpublications.gliomas.2021

Copyright: The Authors.

License: This open access article is licenced under Creative Commons Attribution-NonCommercial 4.0 International (CC BY-NC 4.0) https://creativecommons.org/licenses/by-nc/4.0/ 
continuous feedback on the patient's cognitive status by reducing the risk of negative mapping. This chapter discusses this novel real-time neuropsychological test and presents the cognitive functional dynamics during surgery and recovery brought to light by the testing.

Keywords: awake surgery; brain mapping; direct electrical stimulation; plasticity; real-time neuropsychological testing

\section{INTRODUCTION}

The purpose of brain mapping with direct electrical stimulation (DES) is to monitor brain function during surgery, permitting surgeons to monitor patient's neurological functions and avoid damage to healthy brain tissue, allowing for maximum possible resection (1-4). It is highly reliable for monitoring simple functional structures such as the sensory-motor system. However, with complex brain networks, DES may not be as reliable, in part due to its on-off effect. Often, postoperative neuropsychological deficits arise. Another point worth noting is the risk of seizure when using DES, which limits the number of stimulations. During surgery, surgeons need to know with greatest possible certainty what functional consequence their actions will have. This can come from constant feedback from the patient responding throughout the resection time. In the present chapter, we describe real-time neuropsychological testing (RTNT) which is an intensive, sustained neuropsychological testing method (5) that has been developed as a complement to DES. The advantages and pitfalls of RTNT are also presented.

\section{DIRECT ELECTRICAL STIMULATION}

By using DES, a map is generated with critical points defined as "positive". An electrical stimulation triggers an effect, for instance a speech arrest, or a hand or lip movement. Three subsequent observations showing an effect at a certain site is a "positive" effect, which is sufficient for interrupting surgery. The given site with positive effect is considered functional, and if resected, could have detrimental post-surgical neurological or neuropsychological consequences. From a surgical point of view, the information about positive effect should be constant, maintaining the feedback throughout the procedure. Due to time constraints related to surgery, DES allows for short testing periods, either at cortical or subcortical levels. In addition, between two subsequent DES phases, resection continues. In that time lapse, no feedback on the patient's cognitive status is available. The negative aspect of this on-off effect is that there are frequent cases where DES does not produce a response. In such a "negative mapping" scenario, surgeons are left without any feedback. Several factors can contribute to negative mapping. Due to the focal nature of stimulation exerted, DES can electrically condition only a small and circumscribed area. The probability of obtaining a negative mapping is relatively high for cognitive functions. However, negative mapping does not always imply lack of functional role of the stimulated area and there can be deficits even after a negative response to DES. 


\section{REAL-TIME NEUROPSYCHOLOGICAL TESTING}

Since 2011, the RTNT has been adopted at our Neurosurgery Unit of the Azienda Sanitaria Universitaria del Friuli Centrale as complementary to DES (5). It has been developed by our team to reduce the problems that may occur in cases of negative mapping where, as described above, a function is not detected by DES, but it does not necessarily mean that the stimulated area is not functional. The RTNT is an intensive, continuous enlarged neuropsychological testing performed throughout the surgery (Figure 1). It allows the immediate detection of neuropsychological dysfunction providing a constant and continuous functional feedback of patient's cognitive status to the surgeon, reducing the problem of missing information from DES. The test selection is based on the anatomical location. An important step is that the tests are switched from one to another very quickly to prevent a potential mismatching problem between task and anatomical point, and to maintain a time course similar to the resection times. This is a delicate phase of surgery, highly dependent on the expertise of the neuropsychologist who must be able to choose the most appropriate task for the area. The neuropsychologist has then to evaluate the performance of the patient. The baseline is constituted by the pre-surgical level of performance on the same tests (such as those tapping language, memory, visual-perception, theory of mind, executive functions, visuo-spatial transformation) that are presented also during surgery. The limit used to stop surgery temporarily or definitively coincided with a decrease of patient's performance to $70 \%$ of baseline levels.

With this approach, we observed a new feature-transitory neuropsychological dysfunction-a reduction of the performance which may completely recover to normal levels after several minutes. This sudden oscillatory scoring has never been observed while using DES alone. We argue that this pattern is suggestive of a reversible effect of mechanical tissue manipulation during surgery. Similar oscillatory scoring effects occur during intramedullary surgery (6) or surgery close to the cortico-spinal tract (7). As soon as the patient exhibited a decrement, the neurosurgeon is immediately informed and the surgeon moves resection to another part of the surgical field and return to the same point where resection altered performance after some time, if the patient has recovered.

Moreover, this method provided additional information about the intraoperative level of patients' behavior by adding a new feature that was not observed using DES alone: reversible intra-operative neuropsychological dysfunction, which consists of oscillations in the patient's responses and a richer insight into their ability to recover functionality.

RTNT comprises of a continuously repeated sequence of neuropsychological tests for lexical comprehension, picture naming, repetition, limb apraxia, shortterm memory and reading. Tests are presented in a fixed order. For example, it can start with object naming, then jump to phonemic discrimination, then to word reading, and to word repetition, followed by pseudoword reading, pseudoword repetition, later to phonological discrimination, digit-span, and lastly to lexical decision, and action naming. Each of the test includes a list of 10 stimuli. Once a sequence of test is completed, the sequence is repeated, yet with a different stimulus list (Figure 2). This test repetition and alternation continues until the end of resection. When the patient completes the test at the same level as pre-surgery, resection continues (Figure 3). As soon as the neuropsychologist detects a decrement vs. the $70 \%$ threshold, the neurosurgeon is informed. 


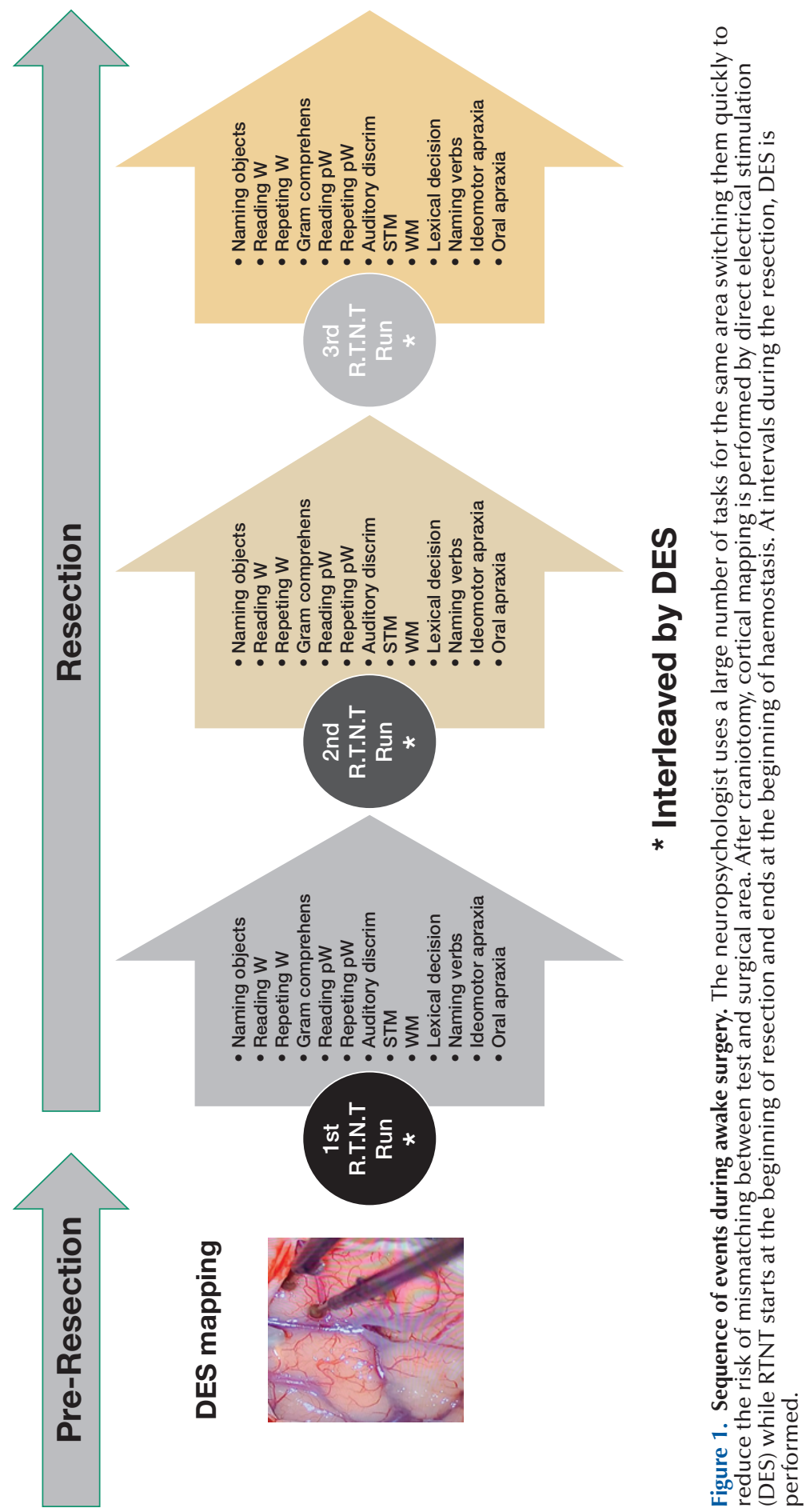


Data from RTNT are interpreted with respect to the patient's correct responses ranging from 0-100\%, compared to their pre-surgery level (RTNT works in percentages, $100 \%$ being optimal brain function). By analyzing a preliminary series of patients, we found that an RTNT deterioration up to $70 \%$ is acceptable because it will allow the patient to make a full recovery. Once the performance is $70 \%$, namely the patient is presented with 10 stimuli of a given test and fails 3 stimuli, performance is calculated vs. $70 \%$ of accuracy. Due to the possibility that it is just a reversible worsening of the performance, before deciding that it is a definitive value, we move surgery to another area and returning to proceed with surgery in the same area only in case the patient has recovered. At intervals, we stopped the procedure to make a further check with DES, but it was negative in the majority of cases, even at the points where a deterioration of the performance has been found.

\section{Validation}

We validated this new approach in 92 patients and the results were published in 2016 (5). RTNT has thus far been performed on a continuous series of about 300 patients and is currently in use at our Neurosurgery Unit of the Azienda Sanitaria Universitaria del Friuli Centrale. The main goal of RTNT is to preserve the patient's cognitive status. This approach has improved the extent of the resection reaching an average of $95 \%$ (range $73 \%-100 \%$ ) while the extent of resection of a previous series of patients operated without using RTNT, which was based only on DES, information reached 90\% (range 49\%-100\%) as shown in Figure 3C. All the data have been recorded and besides the intra-operative quick analysis we do, it has been possible to analyze them for more detailed analyses after surgery, mainly for research purposes, as presented in Figure 3A.

\section{Left and right hemisphere}

A patient undergoing a right insular resection presented with good cognitive performance throughout RTNT but experienced some neurophysiological symptoms such as strange taste and a repeated tendency to fall asleep (8). A patient with a left insular lesion maintained normal language functions but showed neurophysiological symptoms such as pain due to partial seizure arising from the temporo-/ insular area as evidenced by electrocorticography (ECoG).

In the left hemisphere, we reported RTNT testing of reading abilities in a series of 49 patients (9). We found that reading performance decreased across RTNT runs in 18 patients, while accuracy remained above $70 \%$ in the majority of cases. Resections ranged from posterior, inferior and middle temporal gyrus to the temporo-parietal areas and the precentral gyrus. There was a good degree of concordance between intra- and immediate postoperative performance with a predictive value found for RTNT, and we reported spontaneous recovery of the post-surgery reading impairment at follow-up.

In the right hemisphere gliomas $(10,11)$, there are reports of post-surgery neuropsychological deficits. Until recently, the tendency was to operate on patients with right hemisphere lesions under general anesthesia. It is acknowledged that right hemisphere-related functions are important for maintaining a good quality 


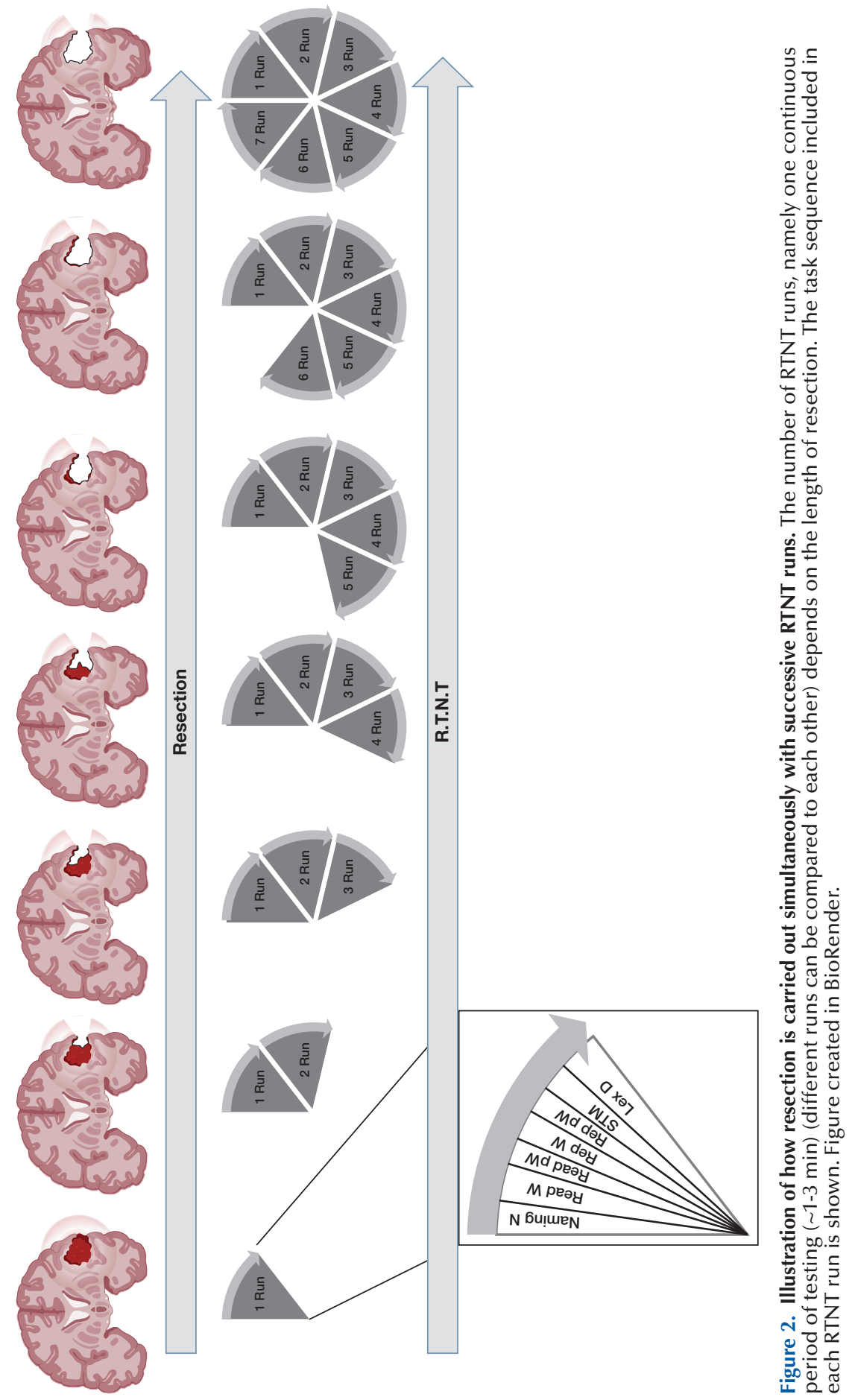




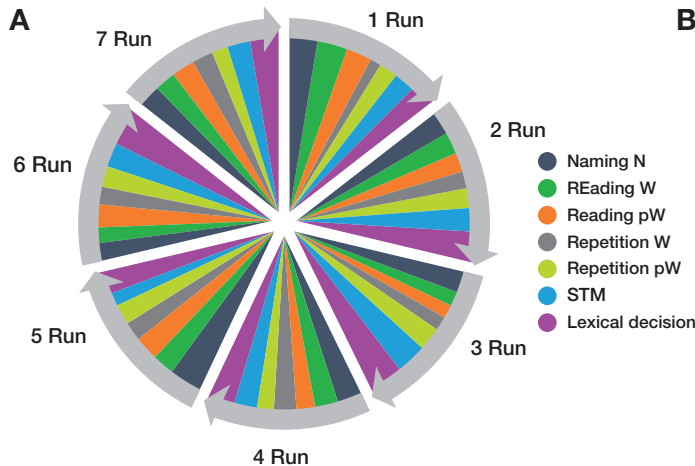

B

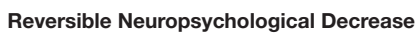

C

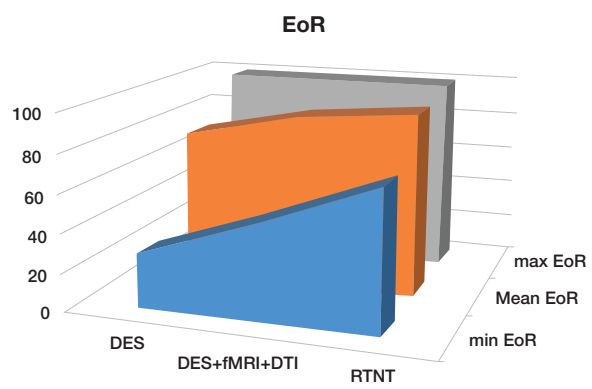

\begin{tabular}{|c|c|c|c|}
\hline & DES & DES+fMRI+DTI & RTNT \\
\hline $\min \mathrm{EoR}$ & 28 & 49 & 73 \\
\hline Mean EoR & 77 & 90 & 95 \\
\hline $\max$ EoR & 100 & 100 & 100 \\
\hline
\end{tabular}

Figure 3. Sequence of RTNT runs and list of tasks. A. The same color represents the same task which is repeated across Runs (yet with different stimuli). B. Patient's performance, expressed in accuracy $(\%)$, shows that for naming and lexical decision tasks there was a reversible neuropsychological decrease around the $3^{\text {rd }}$ Run. We learnt recognizing and detecting reversible neuropsychological deficit patterns, which are often followed by recovery, allowing to proceed with the resection. A positive DES would simply have stopped resection. C. The extent of resection over time evolved and increased when RTNT was introduced.

of life and should be preserved during awake surgery when possible (12). Intraoperative monitoring of higher functions related to the right hemisphere include visuospatial cognition, socio-cognitive functions and executive functions which are still poorly documented and remain an open issue. We adopted four RTNT protocols for the frontal and parietal lobes, the temporo-insular region and premotor area, except for the motor sensory function. Our experience with RTNT in the right hemisphere involves 103 patients. The most frequently used task in patients with lesions involving the visuo-spatial network is the Verbal Milner Landmark task (13) (Figure 4A) which is used to monitor visuo-spatial functions and detect the first signs of neglect. In this task, the patient is required to decide which part of a segment colored in black and red is longer (for half of the items) 
or shorter (for the remaining of the items). The mean accuracy achieved by this group of patients is $80.55 \%$. In summary, RTNT is currently used in selected cases for awake surgery in the right hemisphere as it enables us to try and extend resection based on feedback of a proficient performance.

A new feature we observed during RTNT is behavioral/emotional reactions. In an exploratory analysis performed on 100 patients, we found that these were most frequently observed (Mann-Whitney Test, $Z=-1.97, \mathrm{p}<.05$ ) in patients with right hemisphere lesions compared to patients with left hemisphere lesions. Behaviors or neurophysiological manifestations included panic, complaining, crying, pain, nausea, apathy, anger, agitation, sweating, and excessive tiredness (Figure 4B). We observed that often lesions in such patients are localized in the temporo-insular area (Figure 4C). This did not change the surgical strategy.

A
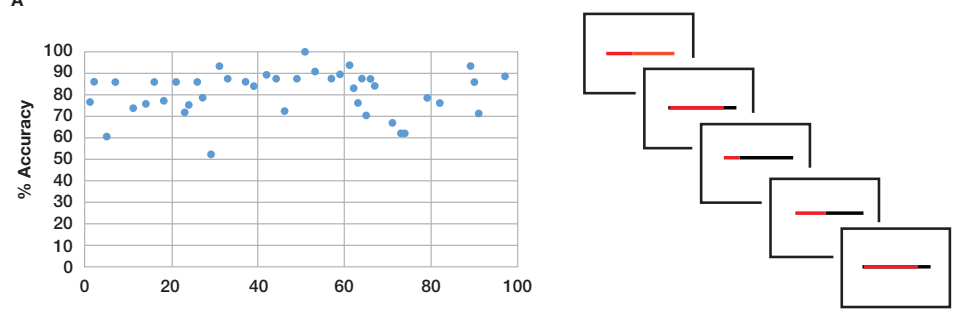

B
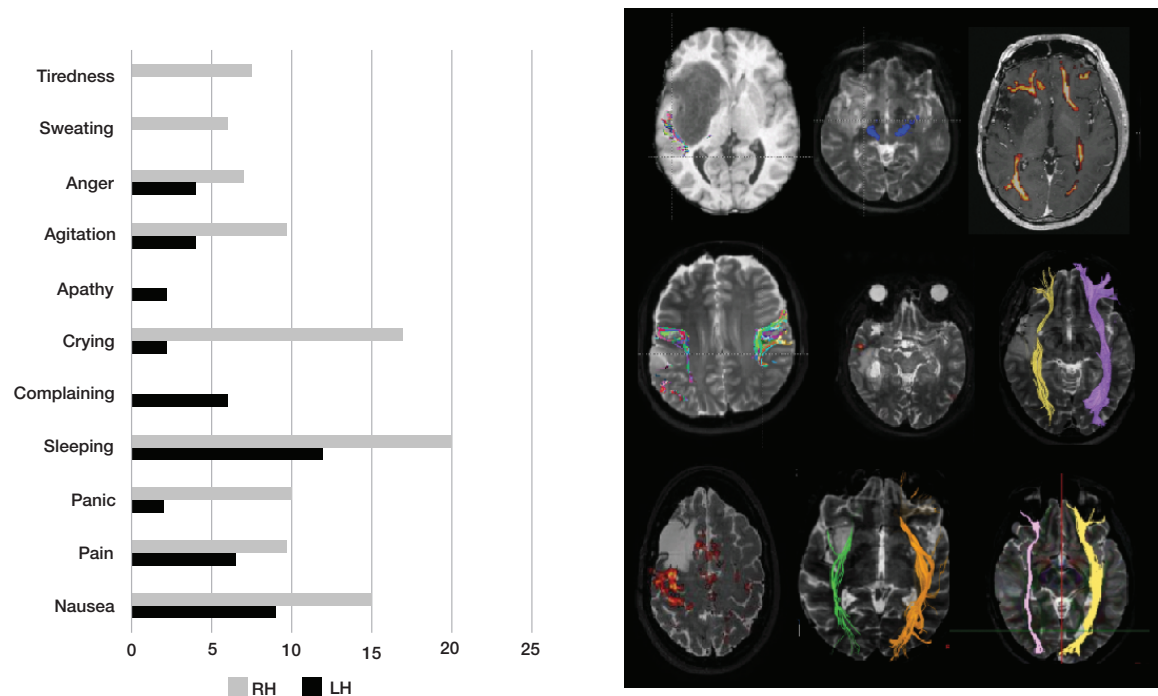

Figure 4. Patients' overall accuracy. A. Milner Landmark Test, and an example of the different items with shorter (black) or longer segments (red). B. Patients (\%) showing behaviors or neurophysiological manifestations, and examples of the lesion localization in patients with right hemisphere lesions showing such phenomena. 


\section{Recurrent surgery}

We retrospectively examined a series of 40 patients undergoing recurrent surgery in the left hemisphere, mainly in the left insular cortex and in the left inferior frontal gyrus (14). The study first compared the pre- and post-surgical neuropsychological status after first and second surgery. We found no significant difference in the number of patients scoring within the normal range. Their level of performance did not differ statistically either. In the same study, we addressed the patients' neuropsychological evolution from pre- and post-resection assessments of the initial and the recurrent surgery and found no significant difference in the number of patients scoring within the normal range. In addition, we analyzed their level of performance, which was stable for all tasks except that for phonological fluency. Based on these results, we argued that repeated glioma surgery is possible without major neuropsychological sequelae.

\section{Young patients}

The RTNT approach has been adapted to awake surgery for young patients. We developed the Junior Real Time Neuropsychological Testing (j-RTNT) for young patients as neuropsychological tests differ from those used with adults. We included neuropsychological tasks related to the area to be resected; the tasks were taken from the neuropsychological assessment for preschoolers, children, and adolescents (Nepsy-II), creating different RTNT runs (15).

\section{Advantages and disadvantages of RTNT}

The RTNT approach offers the possibility to obtain a continuous and reliable feedback about the patient's cognitive status, through all the resection phases. The test administration occurs continually, while DES is discontinuously performed. RTNT is not subjected to the limits of a focal stimulation like DES, thus avoiding the risk of negative mapping. In our experience, RTNT allows a quicker resection, since the surgeon continuously receives feedback on the patient's status. In addition, by using the RTNT approach we detected a new neuropsychological feature, that is the reversible neuropsychological decrease, and we learn by experience that in the majority of the cases, resection could continue. In contrast, a positive DES would stop resection. As a consequence, the extent of resection could be different by using the two approaches. RTNT is designed to include a large number of tests and stimuli, and this property allows a higher confidence and a proper match between an area and the test. By analyzing the patients' follow-up neuropsychological tests, we could learn the most reliable test to be used intra-surgery for a given area, those who fully recover in the post-surgical phase, and those who are more resistant to recovery. We also gained a better knowledge about the tasks that are useful during surgery in the right hemisphere and in general, a better insight in understanding higher level cognition during surgery. Lastly, RTNT made it possible to increase the average extent of resection and the delivery of a continuous feedback on the patient's cognitive status enabling surgeons to achieve gross total (and supra-total) resections. 
The disadvantage of using the RTNT approach is that demanding levels of collaboration and flexibility are required. This can limit the number of tests that can be used intra-surgery. In addition, a high neuropsychological expertise is also required.

\section{CONCLUSION}

The first prerequisite in glioma surgery, particularly for low-grade gliomas, is to remove the tumor as extensively as possible to improve prognosis. Low-grade gliomas suited for surgery are generally located supratentorially involving the cortex and the white matter beneath. Apart from simpler functions, meaning motor and somatosensory functions, which actually are represented in a very limited part of the central area, the remaining cortical areas and the white matter are involved in cognitive functions of varying complexity. These higher cognitive functions are based on complex network systems and as seen clinically, are characterized by high inter-individual variability (16-18). The only way to monitor these functions is neuropsychological testing-aware that the anatomo-functional correlations are often limited with respect to the microsurgical spatial resolution. It is only by using a large number of tests that we can avoid mismatches with the anatomical localization and have a relatively safe functional feedback of the area under resection. Our experience indicates that real-time testing needs patients to be highly collaborative, and this is the only limitation of this approach. This method is demanding for both the neuropsychologist and the patient. For each individual patient, the greater the collaboration during these tests taking place one immediately after the other, the higher the quality of the feedback indicating whether surgery is progressing safely. This approach also offers higher prediction on the recovery of higher cognitive functions.

Conflict of interest: The authors declare no potential conflicts of interest with respect to research, authorship and/or publication of this chapter.

Copyright and permission statement: The authors confirm that the materials included in this chapter do not violate copyright laws. Where relevant, appropriate permissions have been obtained from the original copyright holder(s), and all original sources have been appropriately acknowledged or referenced.

\section{REFERENCES}

1. Ojemann G, Ojemann J, Lettich E, Berger M. Cortical language localization in left, dominant hemisphere. An electrical stimulation mapping investigation in 117 patients. J Neurosurg. 1989;71:316-26. https://doi.org/10.3171/jns.1989.71.3.0316

2. Ojemann GA. Functional mapping of cortical language areas in adults. Intraoperative approaches. Adv Neurol. 1993;63:155-63.

3. Berger MS. Minimalism through intraoperative functional mapping. Clin Neurosurg. 1996;43:324-37.

4. Berger MS, Ojemann GA. Intraoperative brain mapping techniques in neuro-oncology. Stereotact Funct Neurosurg. 1992;58:153-61. https://doi.org/10.1159/000098989 
5. Skrap M, Marin D, Ius T, Fabbro F, Tomasino B. Brain mapping: A novel intraoperative neuropsychological approach. J Neurosurg. 2016;5:1-11. https://doi.org/10.3171/2015.10.JNS15740

6. Sala F, Bricolo A, Faccioli F, Lanteri P, Gerosa M. Surgery for intramedullary spinal cord tumors: the role of intraoperative (neurophysiological) monitoring. Eur Spine J. 2007;16:S130-S139. https://doi. org/10.1007/s00586-007-0423-x

7. Skrap M, Vescovi MC, Pauletto G, Maieron M, Tomasino B, Bagatto D et al. Supratentorial Cavernous Malformations Involving the Corticospinal Tract and Sensory Motor Cortex: Treatment Strategies, Surgical Considerations, and Outcomes. Oper Neurosurg. 2018;15:483-97. https://doi.org/10.1093/ ons/opx281

8. Tomasino B, Marin D, Ius T, Skrap M. Neuropsychology in Insular Lesions Prior-During and After Brain Surgery. In: Turgut M., Yurttaş C., Tubbs R. (eds) Island of Reil (Insula) in the Human Brain. 2018; Springer, Cham. https://doi.org/10.1007/978-3-319-75468-0_31

9. Tomasino B, Ius T, Skrap M, Luzzatti C. Phonological and surface dyslexia in individuals with brain tumors: Performance pre-, intra-, immediately post-surgery and at follow-up. Hum Brain Mapp. 2020;41:5015-31. https://doi.org/10.1002/hbm.25176

10. Klein M, Duffau H, De Witt Hamer PC. Cognition and resective surgery for diffuse infiltrative glioma: an overview. J Neurooncol. 2012;108:309-18. https://doi.org/10.1007/s11060-012-0811-x

11. Herbet G, Lafargue G, Moritz-Gasser S, Menjot de Champfleur N, Costi E, Bonnetblanc F, et al. A disconnection account of subjective empathy impairments in diffuse low grade glioma patients. Neuropsychologia. 2015;70:165-76. https://doi.org/10.1016/j.neuropsychologia.2015.02.015

12. Vilasboas T, Herbet G, Duffau H. Challenging the Myth of Right Nondominant Hemisphere: Lessons from Corticosubcortical Stimulation Mapping in Awake Surgery and Surgical Implications. World Neurosurg. 2017;103:449-56. https://doi.org/10.1016/j.wneu.2017.04.021

13. Capitani E, Neppi-Modona M, Bisiach E. Verbal-response and manual-response versions of the Milner Landmark task: normative data. Cortex. 2000;36:593-600. https://doi.org/10.1016/S0010-9452 (08)70540-9

14. Capo G, Skrap M, Guarracino I, Isola M, Battistella C, Ius T et al. Cognitive Functions in Repeated Glioma Surgery. Cancers. 2020;12:1077. https://doi.org/10.3390/cancers12051077

15. Guarracino I, Ius T, Pauletto G, Maieron M, Skrap M, Tomasino B. Junior-Real Time neuropsychological testing (j-RTNT) for a young patient undergoing awake craniotomy. Brain Cogn. 2020;140:105535. https://doi.org/10.1016/j.bandc.2020.105535

16. Veenith TV, Carter E, Grossac J, Newcombe VFJ, Outtrim JG, Lupson V, et al. Inter Subject Variability and Reproducibility of Diffusion Tensor Imaging within and between Different Imaging Sessions. PLoS ONE. 2013;8:e65941. https://doi.org/10.1371/journal.pone.0065941

17. Chechlacz M, Gillebert CR, Vangkilde SA, Petersen A, Humphreys GW. Structural Variability within Frontoparietal Networks and Individual Differences in Attentional Functions: An Approach Using the Theory of Visual Attention. J Neurosci. 2015;35:10647-58. https://doi.org/10.1523/ JNEUROSCI.0210-15.2015

18. Bernard F, Zemmoura I, Ter Minassian A, Lemée JM, Menei P. Anatomical variability of the arcuate fasciculus: a systematical review. Surg Radiol Anat. 2019;41:889-900. https://doi.org/10.1007/ s00276-019-02244-5 
